\title{
Fabrication of transparent composites for non-invasive Surface Enhanced Raman Scattering (SERS) analysis of modern art works
}

\author{
Abeer Alyami ${ }^{1}$, Antonio Mirabile ${ }^{2}$ and Daniela lacopino ${ }^{1^{*}}$ (D)
}

\begin{abstract}
Surface Enhanced Raman Scattering (SERS) has become an invaluable tool for the identification of colorants in artworks, due to its enhanced sensitivity and ability to quench fluorescence interference compared to Raman spectroscopy. However, the application of SERS to artwork analysis is still limited by its inherent invasiveness and the need for extraction procedures. In this work non-invasive transparent SERS probes were fabricated by self-assembly of Ag nanoparticles into glass and PDMS surfaces and used for identification of dye content in artistic drawings. SERS measurements were performed directly on the selected analytical surfaces by laser back illumination through the SERS probe. The non-invasiveness of fabricated probes was tested by optical microscopy. It was found that Ag nanoparticle/glass probes left no Ag nanoparticle residue after four consecutive depositions on sacrificial surfaces, whereas Ag nanoparticle/PDMS composites could be deposited and subsequently peeled off the analytical surfaces leaving no contamination traces. The high conformability, flexibility and transparency of Ag nanoparticle/PDMS composites enabled good adhesion to the surface of analyzed artistic drawings and therefore the generation of in situ SERS spectra from artistic drawings. The use of this method allowed identification of main dye components in real artworks comprising a red BIC ballpoint drawing and a Japanese woodblock print.
\end{abstract}

Keywords: Raman spectroscopy, SERS, Non-invasive, Paper artwork, BIC ballpoint pens, Japanese wood print

\section{Introduction}

The ability to identify colorants and colorant mixtures in artworks is of fundamental importance to inform application of conservation treatments, display and preservation procedures as well as to allow identification of artworks' provenance and forgery. Due to its inherent non-invasive nature, fingerprinting capability, easiness of use and availability of portable instrumentation, Raman spectroscopy is among the most versatile and used spectroscopic techniques for analysis of artworks [1-3]. However, the low sensitivity and the strong fluorescence interference of colorants in the visible part of the spectrum, have largely limited the application of Raman to the analysis of natural and artificial colorants. In the last 20 years Surface

\footnotetext{
*Correspondence: daniela.iacopino@tyndall.ie

${ }^{1}$ Tyndall National Institute, University College Cork, Dyke Parade, Cork, Ireland

Full list of author information is available at the end of the article
}

Enhanced Raman Scattering (SERS) has been established as powerful tool for the identification and analysis of natural and synthetic colorants used in artworks [4-6]. The high sensitivity, up to $10^{8}-10^{12}$ times the sensitivity of Raman spectroscopy and down to single molecule detection, combined with the retained fingerprinting capabilities of Raman spectroscopy, offers key advantages for the identification of colorants, often present in artworks in small concentrations, mixed with other colorants and embedded in complex matrices [7]. In addition, SERS can effectively quench the fluorescence interference caused by laser illumination, thus overcoming Raman spectroscopy limitations and considerably widening the range of applications in conservation science. Accordingly, SERS has been successfully used for investigations of a large range of heritage materials including archeological textile fibers [6], woodblock prints $[8,9]$ and has enabled identification of synthetic and organic dye content in 
polychromic works of art $[10,11]$, painting samples [12, 13] oil paintings [14] and commercial inks [15]. Another advantage of SERS is the availability of lightweight, sensitive and low cost portable instrumentation, whose use is often mandatory for the analysis of large or unmovable artworks.

SERS experiments are usually performed with the use of colloidal concentrated Ag solutions or with rigid ordered plasmonic substrates. Both methods are inherently invasive as they cannot be used directly on the analytical surface and require the use of harsh extraction methods in order to separate by hydrolysis the colorant from its matrix, before application of Ag colloids or deposition onto Ag rigid SERS substrates [16]. Alternative use of $\mathrm{Ag}$ colloidal pastes has been proposed for in situ, non-hydrolysis SERS measurements [17-19]. This approach consists into deposition of a droplet of concentrated colloidal solution on untreated samples and has been shown particularly effective for the characterization of historical textiles. However, the method is still inherently invasive as direct deposition of $\mathrm{Ag}$ pastes stains the work of art, making necessary the physical removal of analyte fragments and their immobilization onto arbitrary substrates prior Ag paste application.

Consequently, many efforts in the last few years have concentrated on the development of non-invasive SERS substrates. A micro-extraction process was developed by Leona et al. who used a bead of polymer hydrogel loaded with a water/DMF/EDTA mixture to extract minimal amounts of colorants from works of arts [20]. The hydrogel with extracted colorants was then treated with Ag nanoparticles for SERS analysis. Successful demonstration of non-invasive identification of crystal violet on deep purple colored areas of Japanese woodblock prints on paper was obtained by this method. Doherty et al. designed and fabricated an active SERS film constituted by Ag nanoparticles doped into a methylcellulose matrix [21]. The resulting composite was transparent, could be applied to the surface of an artwork and removed after analysis. Strongly enhanced SERS spectra were obtained for powder pigments and lake/egg tempera, otherwise not measurable by Raman spectroscopy. However, close observation of detached composites showed that analytical surface fragments $(40 \mu \mathrm{m})$ underwent removal and trapping into the composite matrix, making the method slightly invasive in nature. One step towards truly nondestructive SERS analysis is the implementation of "dry state" measurements, which has been achieved by immobilization of Ag colloids on the distal end of an optical fiber. In this way Polwart et al. successfully obtained SERS spectra of crystal violet from historical costumes of the late nineteenth century [22]. Stokes et al. fabricated a SERS-inducing nanoprobe by thermally evaporating a thin layer of silver nanoparticles to the tip of a tapered optical fiber (100 nm tip diameter) [23]. Spectra of brilliant cresyl blue coated on a glass slide were recorded by touching dry dye samples with the nanoprobe. Simpler substrates were fabricated by Zaffino et al. by deposition of Ag islands (fabricated by beam evaporation) or Ag colloidal particles (chemically synthesized) over glass slides [24]. Spectra of alizarin, purpurin and lac dye were obtained by deposition of $\mathrm{Ag}$ island substrates on dyed wool threads. However, the authors report that scanning electron microscopy energy dispersive X-ray analysis (SEM-EDX) of post-treated wool threads showed contamination from $\mathrm{Ag}$ islands and colloidal particles. Although residues were small in size $(1-3 \mu \mathrm{m})$, the oxidation of Ag may lead to surface color changes not acceptable for works of art analysis.

The aim of this work was to overcome the limitations above through the fabrication of novel SERS substrates obtained by deposition of self-assembled Ag nanoparticles into glass and PDMS substrates and to demonstrate their application in non-invasive analysis of artworks.

\section{Experimental section \\ Materials and methods}

Silver nitrate, sodium borohydride, trisodium citrate, octadecylamine (ODA), chlorobenzene, methanol, ethanol were purchased from Sigma-Aldrich used without further purification. Reference dyes Crystal violet (CV), Rodhamine B and Blue 38 were also purchased from Sigma-Aldrich. SYLGARD 184 Silicon Elastomer Kit for polydimethylsiloxane (PDMS) film fabrication was also purchased from Sigma-Aldrich. All glassware was cleaned with aqua regia prior to silver nanoparticles synthesis. Milli-Q water (resistivity $418 \mathrm{M} \Omega / \mathrm{cm}$ ) was used throughout the experiments.

Medium and Crystal BIC pens were purchased from local stores. Japanese prints and Anne-Flore Cabanis drawings were kindly donated by paper conservator Antonio Mirabile.

\section{Synthetic procedures \\ Ag nanoparticle synthesis}

Ag nanoparticles were synthesized according to a process reported previously by Rainville et al. [25]. Briefly, $12 \mathrm{~mL}$ of an aqueous solution $(0.2 \% \mathrm{w}: \mathrm{w})$ of silver nitrate and $488 \mathrm{~mL}$ of deionized water were mixed and heated to $100{ }^{\circ} \mathrm{C}$. Next, $11.6 \mathrm{~mL}$ of an aqueous solution of sodium citrate $\left(3.4 \times 10^{-2} \mathrm{M}\right)$ was added, followed $30 \mathrm{~s}$ later by the quick injection of $5.5 \mathrm{~mL}$ of a freshly prepared, icecold aqueous solution of sodium borohydride $(0.2 \mathrm{M})$ and sodium citrate $(0.03 \mathrm{M}, 50 \mathrm{~mL})$. The solution was stirred for $2 \mathrm{~min}$ and subsequently left it to cool at room temperature. The obtained nanoparticle solution was 
centrifuged once at $9000 \mathrm{rpm}$ for $20 \mathrm{~min}$ and redispersed in $4 \mathrm{~mL}$ of distilled water. The concentrated nanoparticle solution was stored in the dark for subsequent use.

Ag nanoparticles were transferred to organic phase by slight modification of a method previously reported [26]. Briefly, $2 \mathrm{~mL}$ of the concentrated aqueous $\mathrm{Ag}$ nanoparticle solution were added to $2 \mathrm{~mL}$ of a $2 \times 10^{-5} \mathrm{M}$ solution of ODA in chlorobenzene. The two solutions were vigorously stirred for $60 \mathrm{~min}$ to facilitate the transfer of $\mathrm{Ag}$ nanoparticles to the organic phase.

\section{Ag nanoparticles/glass/PDMS nanocomposites fabrication}

PDMS was fabricated by pouring 10:1 (w:w) of Sylgard 184 elastomer and curing agent in a petri dish followed by heating at $60^{\circ} \mathrm{C}$ for $24 \mathrm{~h}$. After removal from oven, the PDMS film was rinsed with isopropanol several times, dried with nitrogen gun and cut to small squares of $1 \times 1 \mathrm{~cm}$. The final thickness of the PDMS film was $1 \mathrm{~mm}$.

In order to obtain Ag nanoparticle/glass and PDMS composites, a small aliquot $(10 \mu \mathrm{L})$ of $\mathrm{Ag}$ nanoparticle chlorobenzene solution was deposited on an acetonecleaned glass coverslip. The droplet was left in contact with air (5 min) until $\mathrm{Ag}$ nanoparticles spontaneously self-assembled at the solvent/air interface, as shown by the formation of a metallic lustre layer. A transparent film (either glass or PDMS) was used as support to retrieve the assembled nanoparticles by bringing it into contact with the nanoparticle droplet for $30 \mathrm{~s}$. Excess organic matter was removed by immersing the Ag nanoparticle/ glass/PDMS composite in isopropanol overnight, followed by multiple rinses with fresh isopropanol.

\section{Characterisation}

UV-vis spectra were acquired using an Agilent/HP 8453 UV-vis Spectrophotometer (200 nm to $1100 \mathrm{~nm}$ ). Scanning electron microscopy (SEM) images of Ag nanoparticles and Ag nanoparticle/PDMS composite films were acquired using a field emission (JSM- 6700F, JEOL UK Ltd.) scanning microscope operating at $3 \mathrm{kV}$.

Raman and SERS measurements were obtained from a Renishaw inVia Raman system equipped with a 514 argon ion laser. The laser beam was focused onto the sample through a Leica 20X objective with 0.4 N.A. Acquisition time was usually $10 \mathrm{~s}$ and measured power was $3 \mathrm{~mW}$. Hand held Raman spectra at $785 \mathrm{~nm}$ were obtained from an InPharma spectrometer. The laser power was $50 \mathrm{~mW}$ at sample and acquisition time was between 7 and $20 \mathrm{~s}$.

SERS spectra of reference dyes were taken by back illumination of a slide containing a dried droplet of dye solution $(10 \mu \mathrm{L}, 1 \mathrm{mM}$ in $\mathrm{MeOH})$ with $\mathrm{Ag}$ nanoparticle/ glass composites. SERS measurements of colored papers and paper artworks were performed by gently pressing Ag nanoparticle/glass $(18 \times 18 \mathrm{~mm})$ or Ag nanoparticles/
PDMS composites $(10 \times 10 \mathrm{~mm})$ on the analytical surface (BIC colored paper or paper artwork) for few seconds. SERS signals were collected by back laser illumination. All spectra were background subtracted.

\section{Results and discussion \\ Fabrication and characterization of SERS composites}

Ag nanoparticles used in this study were prepared in aqueous solution by reduction of $\mathrm{Ag}(\mathrm{I})$ salts with strong reducing agent sodium borohydride and subsequently phase transferred in organic solution. SERS probes were constituted by dense layers of Ag nanoparticles immobilized on transparent rigid (glass) and flexible (PDMS) substrates from self-assembled chlorobenzene solutions. The use of organic solutions was motivated by previous studies carried out by our group and others showing that direct deposition of aqueous plasmonic colloidal solutions on glass substrates resulted in dis-homogeneous nanoparticle distribution (i.e. accumulation of nanoparticle along the perimeter of the droplet), due to coffee stain effects [27, 28]. In contrast, homogeneous arrays were obtained by surface transfer of self-assembled superstructures obtained from evaporation of organic droplets. The process of SERS probe fabrication is schematically shown in Fig. 1.

A small droplet $(10 \mu \mathrm{L})$ of concentrated Ag nanoparticle in chlorobenzene solution was deposited on a clean glass substrate and left undisturbed for 5-10 min (1a). Following the evaporation of small amount of solvent, Ag nanoparticles spontaneously self-assembled at the solvent/air interface, as evidenced by formation of a metallic lustre (1b). At this point a receiving substrate (glass or PDMS) was brought in contact with the nanoparticle droplet (1c). Capillary effects promoted the transfer of the metallic layer on the receiving substrate (1d). Following transfer, the glass/PDMS substrate containing Ag nanoparticles was allowed to dry and subsequently immersed overnight in isopropanol to remove unbound particles and excess surfactants present in the organic solution. Figure 1e, f show photographs of $\mathrm{Ag}$ nanoparticles deposited on glass and PDMS substrates, respectively.

Figure 2 show SEM images of (a) as-synthesized Ag nanoparticles in aqueous solutions showing formation of spherical particles with average size of $15 \pm 5 \mathrm{~nm}$ and (b) Ag nanoparticles transferred on glass substrates from self-assembled chlorobenzene droplets, following the method described in Fig. 1. The images showed that particles retained their spherical size through the phase transfer/self-assembly/substrate transfer process and that the final average size of SERS substrate deposited particles was $20 \pm 3 \mathrm{~nm}$. More details on UV-vis response and 


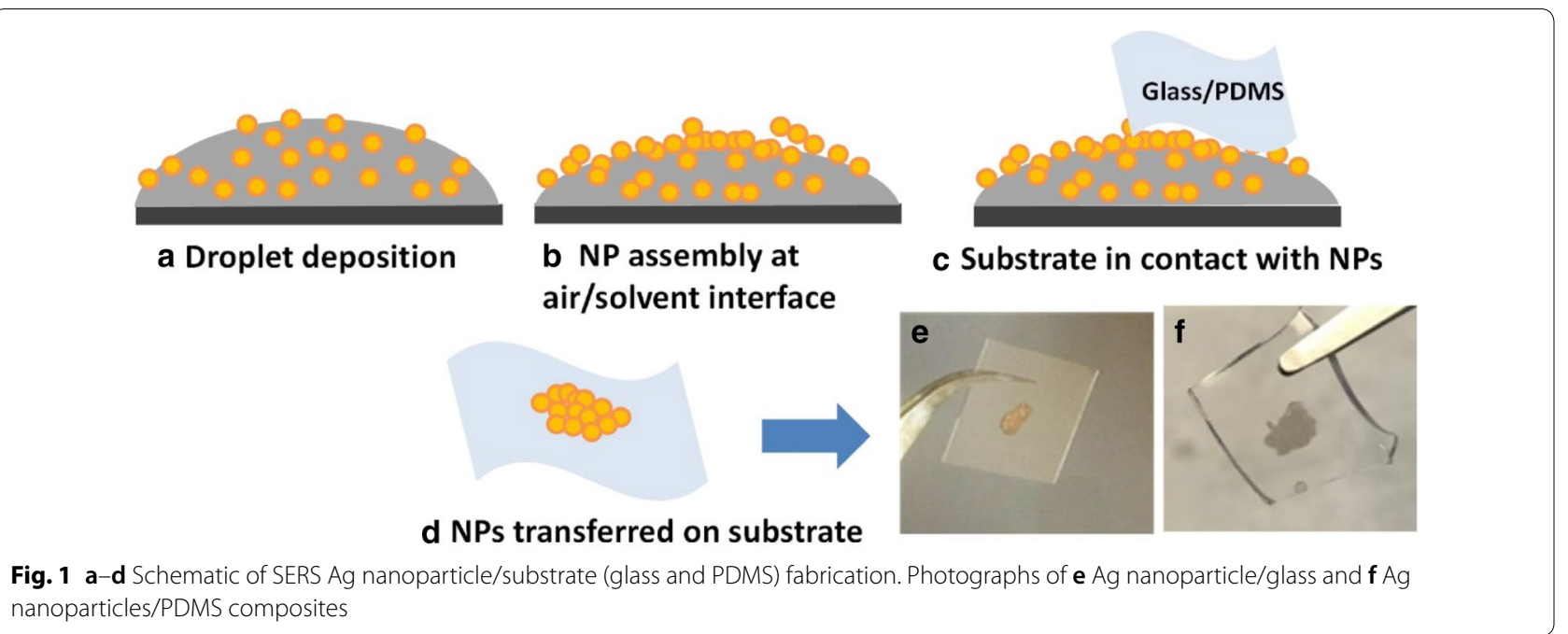

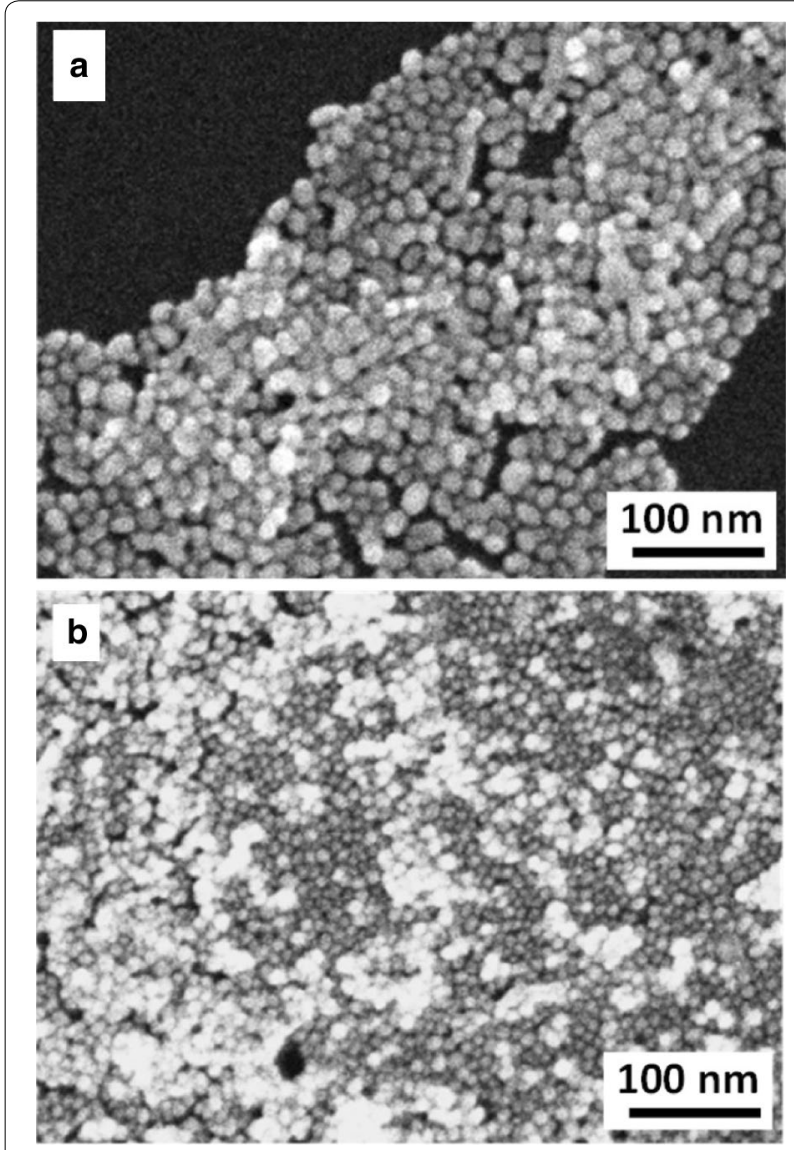

Fig. 2 SEM images of Ag nanoparticles transferred on a glass substrate and $\mathbf{b}$ PDMS substrate from self-assembled chlorobenzene droplets size distribution are reported in Additional file 1: Figures S1, S2).

\section{Minimal invasiveness tests}

Prior attempting SERS analysis on real works of art tests were performed in order to establish the capability of fabricated SERS structures for minimal-invasive analysis. To this end, $\mathrm{Ag}$ nanoparticles/glass and $\mathrm{Ag}$ nanoparticle/PDMS substrates were repetitively placed for few minutes on marked areas of commercial white paper sheets. Close contact between the Ag nanoparticle/glass substrates and the analytical white paper sheet was obtained by placing two glass slides on the sides of the SERS substrate (see Fig. 4a). During SERS measurements this procedure ensured that the substrate was held in place and also maintained close contact between the Ag nanoparticle moiety and the analytical surface necessary for the SERS effect to occur. Optical microscopy images of white paper sheets were taken prior SERS probe deposition (Fig. 3a) and after each deposition (Fig. 3b-f) to assess the accidental release of loosely bound nanoparticles on the analytical surface. As shown by Fig. 3b, the first Ag nanoparticle/glass substrate placement (deposition 1) on the paper substrate left nanoparticle residues; Fig. 3 shows that four repetitive placements had to be performed before no residual traces of Ag nanoparticles could be detected on the paper sheet. In contrast, when Ag nanoparticle/ PDMS substrates were placed on the paper no residual traces of Ag nanoparticles were observed already after the first placement (Fig. 3g, h). It is important to stress 

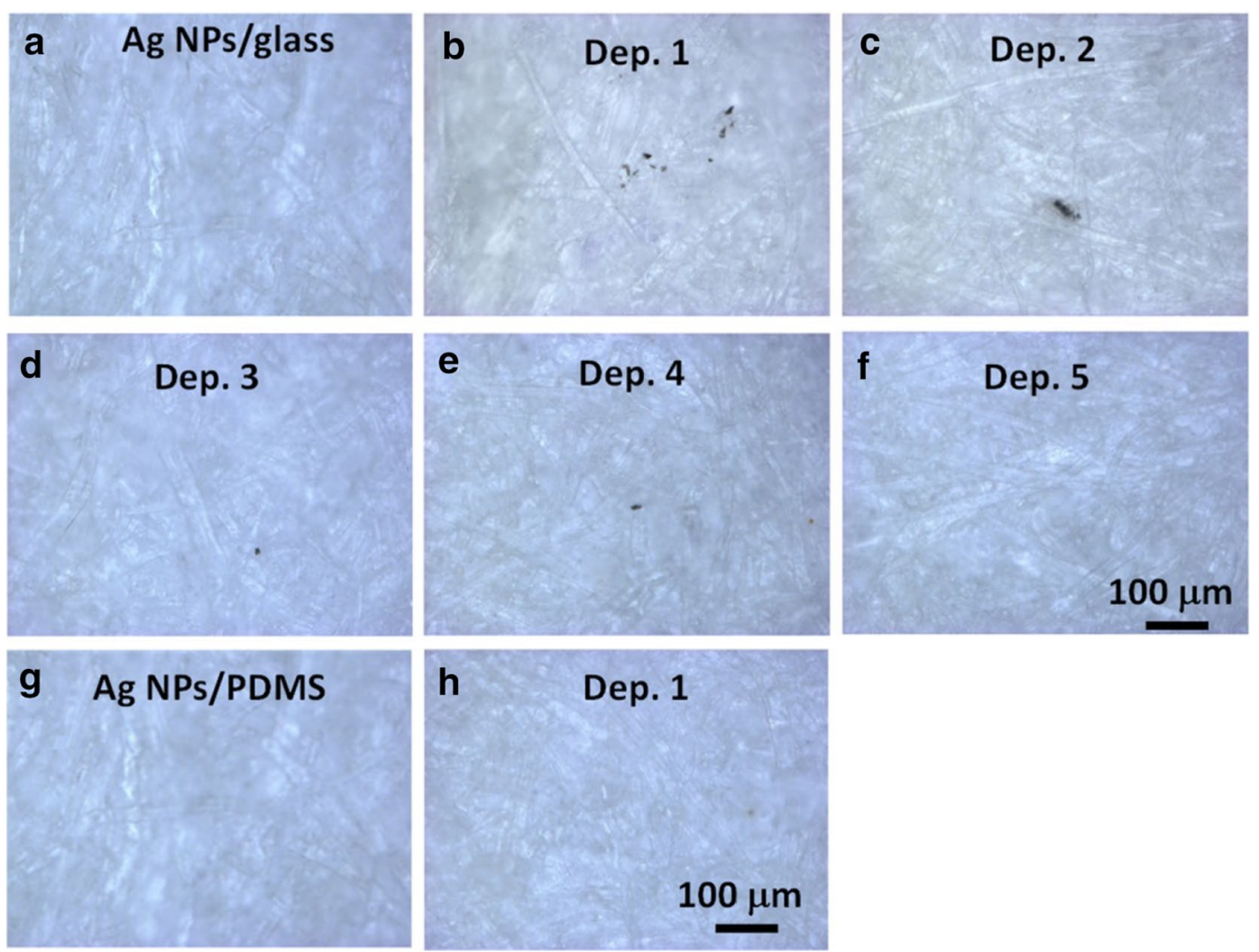

Fig. 3 a Photograph of Ag nanoparticle/glass SERS substrate; $\mathbf{b}-\mathbf{f}$ optical microscopy images of a white paper sheet taken after consecutive depositions of the same Ag nanoparticle/glass SERS substrate showing presence of Ag nanoparticle residues for the first four depositions; $\mathbf{g}$ photograph of Ag nanoparticle/PDMS SERS substrate; $\mathbf{h}$ optical microscopy image of a white paper sheet taken after deposition of Ag nanoparticle/ PDMS SERS substrate showing no trace residues of Ag nanoparticles

that Ag nanoparticle/PDMS substrates were applied to the analytical surface just by slight pressure. The substrates showed good adhesion with the paper surfaces and, in contrast with the Ag nanoparticle/glass substrates, did not require the use of additional weights to hold the SERS substrate in place or to maximize SERS effects. These data show that the adhesion of Ag nanoparticles to PDMS was stronger than the adhesion to glass substrates. One reason could be the strong hydrophobic affinity between PDMS and the hydrophobic ODA molecules crafted on the surface of Ag nanoparticles following their phase transfer in chlorobenzene. It should be pointed out that the strong adhesion of the Ag nanoparticle/PDMS composite to the paper analytical surface was enough to ensure close contact between the Ag nanoparticles and the analytical surface to promote the SERS effect. This effect was due to the use of PDMS and was promoted by the PDMS/
Ag nanoparticle area ratio in the composite. When transferred on PDMS the Ag nanoparticles covered an approximately circular area of $5 \mathrm{~mm}$ in diameter. However, the size of the used PDMS was larger (ca. four times in size). That means that the adhesion to the substrate was not provided by the nanoparticle area but by the PDMS area, which stuck to the surface and ensured adhesion of the overall composite.

\section{SERS tests with Ag/PDMS composites}

The capability of Ag nanoparticle/PDMS composites to perform SERS analysis was initially assessed by taking SERS spectra of model molecule 4-aminobenzene thiol (4-ABT). This particular molecule was selected the occurrence of a SERS effect is easily assessed by the occurrence of additional $b_{2}$ in plane, out of phase vibrational modes not present in the Raman spectrum and attributed to a SERS chemical enhancement effect (plasmonic metal-molecule charge transfer process) [29]. The spectrum of 4-ABT was taken by placing the composite on a dry droplet $\left(1 \times 10^{-6} \mathrm{M}, \mathrm{MeOH}\right)$ of 4 - $\mathrm{ABT}$ on a glass 

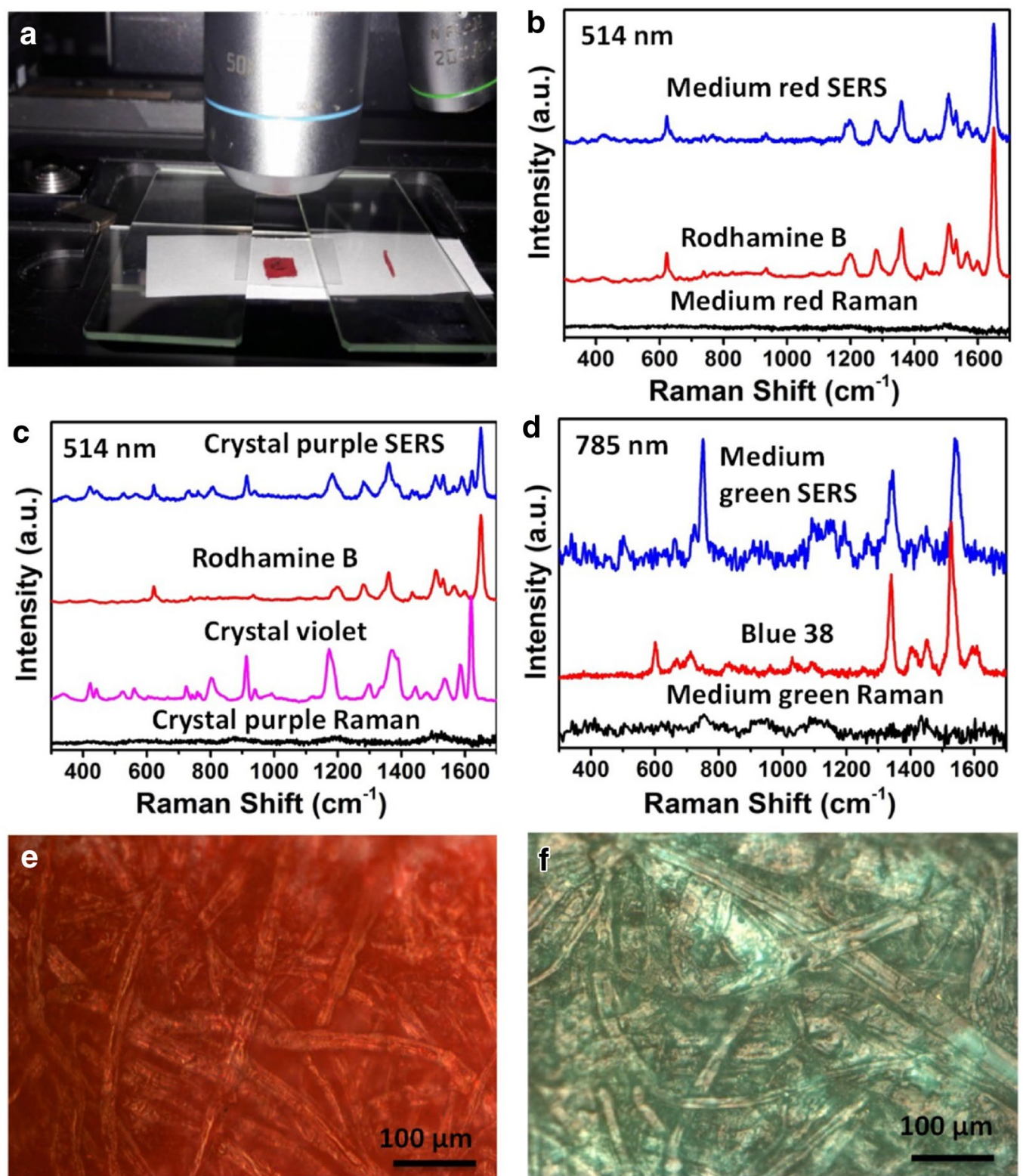

Fig. 4 a Photograph of set up used for SERS analysis of colored paper with Ag nanoparticle/glass SERS substrates; b SERS (blue line) and Raman (black line) spectra of BIC medium red pen square on paper, SERS spectrum of reference Rodhamine B dye (red line). Spectra were taken with bench Raman instrumentation at $514 \mathrm{~nm}$ illumination; c SERS (blue line) and Raman (black line) spectra of BIC crystal purple pen square on paper, SERS spectra of reference Rodhamine B (red line) and Crystal violet (pink line) dyes, 514 illumination; d SERS (blue line) and Raman (black line) spectra of BIC medium green pen square on paper, SERS spectrum of reference Blue38 dye (red line). Spectra taken with handheld Raman instrumentation at $785 \mathrm{~nm}$ illumination; e-f optical images of colored red and green colored squares after SERS analysis

slide with the Ag nanoparticle facing the 4-ABT. SERS spectra were obtained by back laser illumination and showed characteristics $a_{1}$ and $b_{2}$ modes of 4-ABT (see Additional file 1: Figure $S 5$ for detailed description of the 4-ABT SERS spectrum obtained with Ag nanoparticle/ PDMS composites).
SERS analysis on model ink-colored paper samples

In parallel, capabilities of fabricated substrates for in situ SERS analysis of commercial BIC ballpoint inks were tested. All spectra were taken in situ, by applying the Ag nanoparticle/glass substrates on pen colored squares with the Ag nanoparticle facing the analytical surface followed by direct back laser illumination (see Fig. 4a for 
photographic details of the setup). All spectra were background subtracted. Figure $4 \mathrm{~b}-\mathrm{d}$ show Raman and SERS spectra of colored squares drawn on commercial paper by Medium red, Crystal purple and Medium green BIC ballpoint pens, respectively. A bench size Raman spectrograph with $514 \mathrm{~nm}$ excitation wavelength was used for analysis of the red and purple squares. Both colored squares showed featureless Raman spectra saturated by fluorescence. The generation of high fluorescence interference was not surprising as UV-vis spectra (see Additional file 1: Figure S3) showed that both red and purple colored inks were characterized by strong absorption centered at $554 \mathrm{~nm}$, close to the selected excitation wavelength of $514 \mathrm{~nm}$, which resulted in concomitant generation of interference fluorescence emission, and consequent masking of Raman signals. In contrast, the SERS spectra of red and purple colored squares showed dramatically enhanced spectral features (Fig. 4b, c blue lines). The red square showed clear presence of Rodhamine B (Pigment Violet 1, C.I. 45,170), evidenced from the presence of the following vibrational bands: 1647 ( $\mathrm{C}-\mathrm{C}$ bending and $\mathrm{C}=\mathrm{C}$ stretching of xanthene aromatic ring), 1530, 1505 (aromatic C-H bending), 1398-1278 (aromatic $\mathrm{C}-\mathrm{C}$ bending), and 620 (xanthene ring puckering) $\mathrm{cm}^{-1}$ [30]. Rhodamine $B$ and/or rhodamine $6 G$ had been previously identified in red ballpoint pen formulations through Easy Ambient Sonic-spray Ionization Mass Spectrometry (EASI-MS) analysis [31], and Laser Desorption/Ionization Time of Flight Mass Spectrometry (LDI-TOF-MS) [32]. Interestingly, while clear distinction between Rhodamine B and Rhodamine 6G could not been achieved with mass spectrometry techniques, the reported SERS analysis clearly showed presence of Rhodamine B in this particular Medium red BIC ink formulation (Fig. 4c, blue line). The SERS spectrum of the Crystal purple square displayed spectral features similar to the red square (main bands at 1650, 1505 and 1358 and $620 \mathrm{~cm}^{-1}$ ), therefore also suggesting presence of Rhodamine $B$ in the ink mixture. However, also small additional bands centered at 1623 (stretching of benzene rings), 938, 914 (asymmetric stretching and bending of $\mathrm{C}-\mathrm{C}_{\text {center }}-\mathrm{C}$ bonds), 565, 525, 443 and 420 (bending of the $\mathrm{C}-\mathrm{N}-\mathrm{N}$ bonds) $\mathrm{cm}^{-1}$ were observed, indicative of the presence of triarylmethane dye crystal violet (Methyl violet 10B, CI 42,555 ) in the ink mixture [33]. For comparison reference spectra of Rodhamine B and crystal violet are shown in Fig. 4b. The presence of crystal violet was further confirmed by the intense purple color of the ink, its occurrence in blue and black BIC pen ink formulations, and by previous Raman analysis performed on the Crystal purple ink at other excitation wavelengths [19]. It is important to stress that the blank SERS spectrum recorded by placing a Ag nanoparticle/glass substrate on a white paper substrate was featureless and did not display any spectral feature attributable to the excess of stabilizing agents used to promote Ag nanoparticle phase transfer from aqueous to chlorobenzene phases (see Additional file 1: Figure S4).

Previous Raman analysis carried out on colored BIC pens showed that green inks possess good Raman response at $514 \mathrm{~nm}$ illumination wavelengths, making the application of SERS not necessary for identification and analysis [19]. However, in view of analytical restrictions imposed by the preciousness and limited mobility of works of art, often requiring the use of portable instrumentation, it is important to assess the capabilities of low-cost handheld Raman instrumentation for analysis of artistic drawings. Figure 4c shows the Raman and SERS spectra of a green colored square measured with a handheld Raman spectrograph with excitation at $785 \mathrm{~nm}$. The Raman spectrum of the Medium green pen ink resulted featureless. However, the SERS spectrum showed strong enhanced spectral features with peaks centered at 1539, 1342, 758 and $499 \mathrm{~cm}^{-1}$. From previous work carried out in our group, it is known that green inks are often constituted by a mixture of blue and yellow dyes and that blue phthalocyanine Blue 38 dye is used in BIC pen formulations [15]. In this case, identification of the yellow component could not be achieved with portable instrumentation. However, the presence of phthalocyanine Blue 38 was confirmed from peaks at 1344 and $1454 \mathrm{~cm}^{-1}$, which were both attributed to internal vibrations of copper phthalocyanine $(\mathrm{CuPh})$ macrocycle [19]. Figure $4 \mathrm{e}$, $\mathrm{f}$ show optical images of colored papers taken after removal of SERS probes following analysis showing that no residue was left on the analytical surface.

\section{SERS analysis on real works of art}

Finally, Ag nanoparticle/glass and Ag nanoparticle/ PDMS composites were used for SERS analysis of real works-of-art. Figure 5a show photograph of a drawing made in red BIC ballpoint pen by contemporary French artist Anne-Flore Cabanis. The peculiarity of the drawing was its overall circular pattern constituted by one continuous line bent at $90^{\circ}$ angles. As the pen was never lifted from the paper during the drawing, the continuous line showed areas of high ink accumulation, generated by the occasional harder pressure of the pen on the paper at resting and corner points, respectively. In order to maximize spectral response, the laser was focused on one of these high ink concentration areas for SERS analysis. SERS spectra were taken in situ, by applying the Ag nanoparticle/PDMS substrate on the drawing with the Ag nanoparticle facing the analytical surface followed by direct back laser illumination. The SERS spectrum of Cabanis' red drawing is shown in Fig. 5b and was characterized 

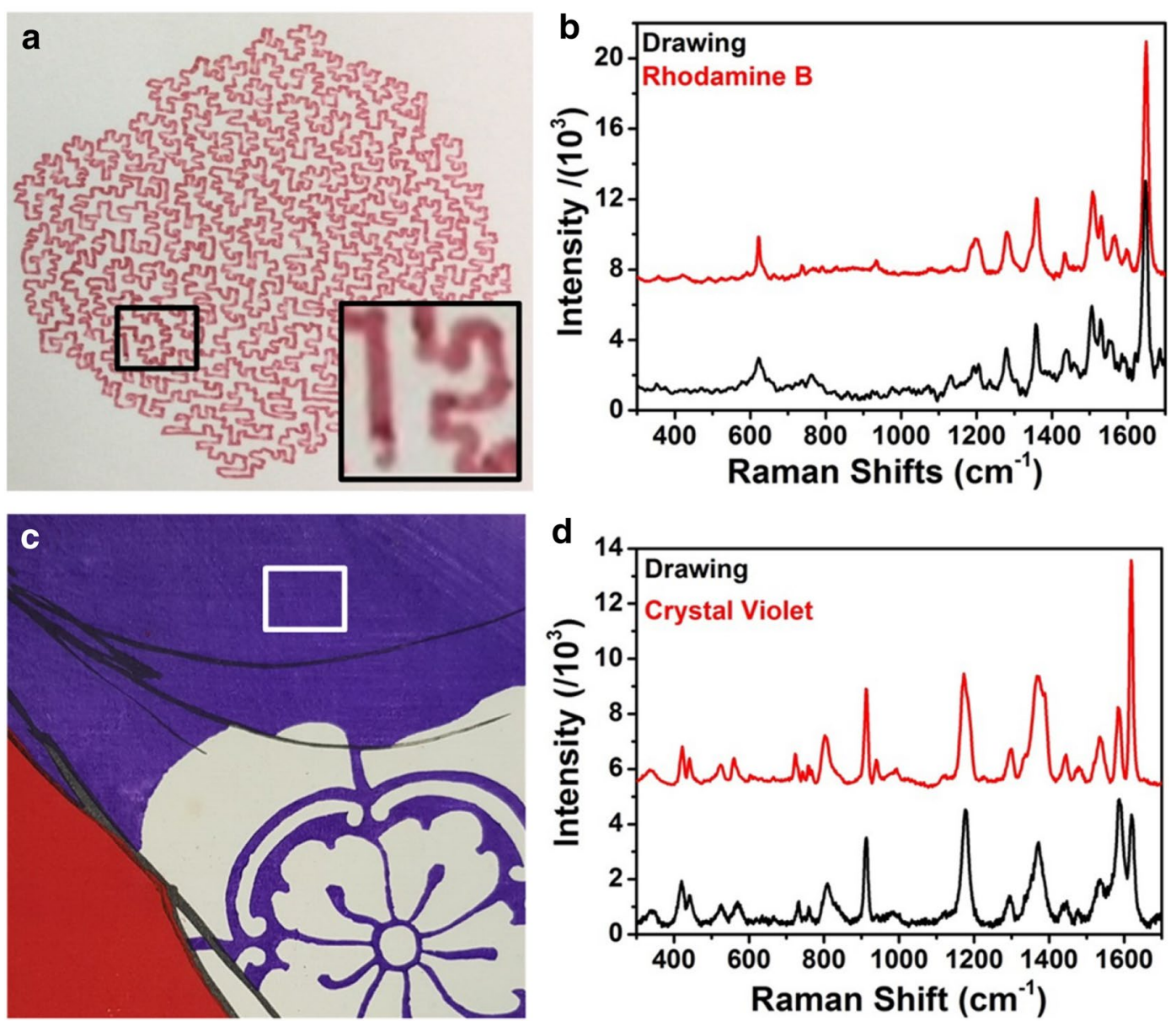

Fig. 5 a, c Photographs of red BIC pen drawing and Japanese wood print analyzed by Ag nanoparticle/PDMS composites. Insets: areas probed by SERS; $\mathbf{b}$ SERS spectra of the red ink drawing showed in a and reference dye Rodhamine B); $\mathbf{d}$ SERS spectra of the purple area showed in $\mathbf{c}$ and reference dye Crystal violet

by one strong band at $1648 \mathrm{~cm}^{-1}$ and medium intensity bands at 1532,1505, 1429, 1352, 1282, 1198 and $623 \mathrm{~cm}^{-1}$ all attributed to Rodhamine B. Figure $5 \mathrm{c}$ shows a photograph of a Japanese wood print characterized by areas of strong purple and red colorations. Figure $5 \mathrm{~d}$ show SERS spectrum of the Japanese print marked purple area recorded by application of Ag nanoparticle/PDMS substrate followed by in situ back laser illumination. The SERS spectrum showed clear bands attributable to Crystal violet at 1622 and $1587 \mathrm{~cm}^{-1}$ (stretching of benzene rings), 1171 and $912 \mathrm{~cm}^{-1}$ (asymmetric stretching and bending of $\mathrm{C}-\mathrm{C}_{\text {center }}-\mathrm{C}$ bonds), 442 and $419 \mathrm{~cm}^{-1}$ (bending of the $\mathrm{C}-\mathrm{N}-\mathrm{N}$ bonds). Rodhamine $\mathrm{B}$ and Crystal violet reference spectra were also added to Fig. $5 \mathrm{~b}$, d, respectively.

\section{Conclusions}

Non-invasive SERS substrates were fabricated by transfer of self-assembled Ag nanoparticles on transparent substrates (glass and PDMS). The deposition of such SERS substrates on analytical surfaces allowed in situ acquisition of SERS spectra by laser back illumination directly on the investigated object without any need for microextraction steps. In contrast to featureless Raman spectra, high intensity and diagnostic SERS spectra were collected for drawings made by red, green and purple BIC ballpoint pens. Following tests ensuring the minimal invasiveness of developed probes, flexible and transparent Ag nanoparticle/PDMS composites were applied to real works of art and SERS spectra obtained for red and purple dyes. The use of SERS has been limited to the necessity for micro-extraction steps, due to the impossibility to deposit SERS probes directly on the analytical surface. As well as transparency, Ag nanoparticle/PDMS composites displayed good adhesion to analytical surfaces and were 
used for the in situ analysis of a modern art drawing and a Japanese woodblock print on paper. Compatibility with both bench and handheld Raman instrumentations was showed, suggesting the potential for Ag nanoparticle/ PDMS composites to be used in the future for analysis of irregular surfaces as well as direct application to flat vertical surfaces. These results further promote the applicability of SERS as highly diagnostic tool for conservation science The high transparency, conformability and flexibility of developed SERS probes allows their deposition on arbitrary and irregular surfaces thus substantially widening the range of SERS applications for analysis and preservation of art works.

\section{Supplementary information}

Supplementary information accompanies this paper at https://doi. org/10.1186/s40494-019-0332-4.

Additional file 1. Characterisation of Ag nanoparticles, UV-vis spectra of BIC pens and reference dyes, Blank SERS spectra, SERS spectrum of 4-aminobenzenethiol.

\section{Abbreviations}

4-ABT: 4-aminobenzene thiol; CuPh: copper phthalocyanine; CV: crystal violet; LDI-TOF-MS: Laser Desorption/lonization Time of Flight Mass Spectrometry; MeOH: methanol; ODA: octadecylamine; PDMS: poly(dimethylsiloxane); SEM: scanning electron microscope; SERS: Surface Enhanced Raman Scattering; EASI-MS: Easy Ambient Sonic-spray Ionization Mass Spectrometry.

\section{Authors' contributions}

AA has performed all experimental work; AM has provided the artwork and supported spectral interpretation; DI has written the manuscript. All authors read and approved the final manuscript.

\section{Funding}

This publication has emanated from research conducted with the financial support of the European Union H2020 project Nanorestart (646063) project.

\section{Availability of data and materials}

All data generated or analyzed during this study are included in this published article and its supplementary information files.

\section{Competing interests}

The authors declare that they have no competing interests.

\section{Author details}

${ }^{1}$ Tyndall National Institute, University College Cork, Dyke Parade, Cork, Ireland.

${ }^{2}$ Mirabile, 11 Rue de Bellefond, 75009 Paris 09, France.

Received: 3 September 2019 Accepted: 18 October 2019

Published online: 31 October 2019

\section{References}

1. Casadio F, Daher C, Bellot-Gurlet L. Raman spectroscopy in archeology and art history. Top Curr Chem. 2016;374:62. https://doi.org/10.1007/ s41061-016-0061-z.

2. Ropret P, Madariaga JM. Applications of Raman spectroscopy in art and archaeology. J Raman Spectrosc. 2014;45:985-92. https://doi. org/10.1002/jrs.4631.

3. Vandernabeele P, Edwards HGM, Moens L. A decade of Raman spectroscopy in art and archeology. Chem Rev. 2007;107:675-86. https://doi. org/10.1021/cr068036i.
4. Roh JY, Matecki MK, Svoboda SA, Wusthotlz KL. Identifying pigment mixtures in art using SERS: a treatment flowchart approach. Anal Chem. 2016;88:2028-32. https://doi.org/10.1021/acs.analchem.6b00044.

5. Brosseau CL, Rayner KS, Casadio F, Grywacz CM, Van Duyne RP. SurfaceEnhanced Raman Spectroscopy: a direct method to identify colorants in various artist media. Anal Chem. 2009;81:74443-7447. https://doi. org/10.1021/ac901219m.

6. Platania E, Lofrumento C, Lottini I, Azzaro E, Ricci M, Becucci M. Tailored micro-extraction method for Raman/SERS detection of indigoids in ancient textiles. Anal Bioanal Chem. 2015;407:6505-14. https://doi. org/10.1007/s00216-015-8816-X.

7. Whitney AV, Casadio F, Van Duyne RP. Identification and characterization of artists' red dyes and their mixtures by surface-enhanced Raman spectroscopy. Appl Spectrosc. 2007;61:994-1000. https://doi. org/10.1366/000370207781745838.

8. Vermeulen M, Leona M. Evidence of early amorphous arsenic sulfide production and use in Edo period Japanese woodblock prints by Hokusai and Kunisada. Herit Sci. 2019;7:73. https://doi.org/10.1186/s4049 4-019-0318-2.

9. Brosseau CL, Casadio F, Van Duyne RP. Revealing the invisible:using surface-enhanced raman spectroscopy to identify minute remnants of color in Winslow Homer's colorless skies. J Raman Spectrosc. 2011;42:1305-10. https://doi.org/10.1002/jrs.2877.

10. Cesaratto A, Leona M, Pozzi F. Recent advances on the analysis of polychrome works of art: SERS of synthetic colorants and their mixtures with natural dyes. Front Chem. 2019;7:105. https://doi.org/10.3389/fchem 2019.00105.

11. Ricci M, Trombetta E, Castellucci E, Becucci M. On the SERS quantitative determination of organic dyes. J Raman Spectrosc. 2018:49:997-1005. https://doi.org/10.1002/jrs.5335.

12. Pozzi F, Porcinai S, Lombardi JR, Leona M. Statistical methods and library search approaches for fast and reliable identification of dyes using surface-enhanced Raman spectroscopy (SERS). Anal Methods. 2013;5:4205-12. https://doi.org/10.1039/C3AY40673C.

13. Zaffino C, Passaretti A, Poldi G, Fratelli M, Tibiletti A, Bestetti R, Saccani I, Guglielmi V, Bruni S. A multi-technique approach to the chemical characterization of colored inks in contemporary art: the materials of Lucio Fontana. J Cult Herit. 2017;23:87-97. https://doi.org/10.1016/j.culhe r.2016.09.006

14. Burke SN, Farling CG, Svoboda SA, Wustholtz KL. Research with undergraduates at the intersection of chemistry and art: Surface-Enhanced Raman Scattering studies of oil paintings. In: ACS Symposium Series, vol. 1305. Chapter 10, 1305. https://doi.org/10.1021/bk-2018-1305.ch010.

15. Alyami A, Saviello D, McAuliffe MAP, Mirabile A, Lewis L, lacopino D. Metal nanoinks as chemically stable surface enhanced scattering (SERS) probes for the analysis of blue BIC ballpoint pens. Phys Chem Chem Phys. 2017;19:14652-8. https://doi.org/10.1039/C7CP01983A.

16. Leona M, Stenger J, Ferloni E. Application of surface-enhanced Raman scattering techniques to the ultrasensitive identification of natural dyes in works of art. J Raman Spectrosc. 2006;37:981. https://doi.org/10.1002/ jrs.1582.

17. Idone A, Gulmini M, Henry Al, Casadio F, Chang L, Apollonia L, Van Duyne RP, Shah NC. Silver colloidal pastes for dye analysis of reference and historical textile fibers using direct, extractionless, non-hydrolysis surfaceenhanced Raman spectroscopy. Analyst. 2013;138:5895-903. https://doi. org/10.1039/C3AN00788J.

18. Wustholz KL, Brosseau CL, Casadio F, Van Duyne RP. Surface-enhanced Raman spectroscopy of dyes: from single molecules to the artists' canvas. Phys Chem Chem Phys. 2009;11:7350-9. https://doi.org/10.1039/B9047 33F.

19. Alyami A, Barton K, Lewis L, Mirabile A, lacopino D. Identification of dye content in colored $\mathrm{BIC}$ ballpoint pen inks by Raman spectroscopy and surface-enhanced Raman scattering. J Raman Spectrosc. 2019;50:115-26. https://doi.org/10.1002/jrs.5512.

20. Leona M, Decuzzi P, Kubic TA, Gates G, Lombardi JR. Nondestructive identification of natural and synthetic organic colorants in works of art by surface enhanced Raman scattering. Anal Chem. 2011;83:3990-3. https:// doi.org/10.1021/ac2007015.

21. Doherty B, Brunetti G, Sgamellotti A, Miliani C. A detachable SERS active cellulose film: a minimally invasive approach to the study of painting 
lakes. J Raman Spectrosc. 2011;42:1932-8. https://doi.org/10.1002/ jrs.2942.

22. Polwart E, Keir RL, Davidson CM, Smyth W, Sadler DA. Novel SERS-active optical fibers prepared by the immobilization of silver colloidal particles. Appl Spectrosc. 2000;54:522.

23. Stokes DL, Chi ZH, Vo-Dinh T. Surface-enhanced-Raman-scattering-inducing nanoprobe for spectrochemical analysis. Appl Spectrosc. 2004;58:292. https://doi.org/10.1366/000370204322886636.

24. Zaffino C, Ngo HT, Register J, Bruni S, Vo-Dinh T. "Dry-state" surfaceenhanced Raman scattering (SERS): toward non-destructive analysis of dyes on textile fibers. Appl Phys A. 2016;122:707. https://doi.org/10.1007/ s00339-016-0209-2.

25. Rainville L, Carolan D, Coelho Varela A, Doyle H, Sheehan D. Proteomic evaluation of citrate-coated silver nanoparticles toxicity in Daphnia magna. Analyst. 2014;139:1678-86. https://doi.org/10.1007/s0033 9-016-0209-2.

26. Alyami A, Quinn AJ, lacopino D. Flexible and transparent Surface Enhanced Raman Scattering (SERS)-active Ag nanoparticle/PDMS composites for in situ detection of food contaminants. Talanta. 2019;201:5864. https://doi.org/10.1016/j.talanta.2019.03.115.

27. Deegan RD, Bakajin O, Dupont TF, Huber G, Nagel SR, Witten TA. Capillary flow as the cause of ring stains from dried liquid drops. Nature. 1997;389:827-9.

28. Deegan RD. Pattern formation in drying drops. Phys Rev E. 2000;61:475-85.
29. Martín A, Pescaglini A, Schopf C, Scardaci V, Coull R, Byrne L, lacopino D. Surface-Enhanced Raman Scattering of 4-aminobenzenethiol on Au nanorod ordered arrays. J Phys Chem C. 2014;118:13260-7. https://doi. org/10.1021/jp503036y.

30. Lin S, Hasi WLJ, Xiang L, Han S, Lou X, Yang F, Lin DY, Lu ZW. Rapid and sensitive SERS method for determination of Rhodamine B in chili powder with paper-based substrates. Anal Methods. 2015;7:5289-94. https://doi. org/10.1039/C5AY00028A.

31. Lalli PM, Sanvido GB, Garcia JS, Haddad R, Cosso RG, Maia DRJ, Zacca JJ, Maldarnec AO, Eberlin MN. Fingerprinting and aging of ink by easy ambient sonic-spray ionization mass spectrometry. Analyst. 2010;135:745-50. https://doi.org/10.1039/b923398a.

32. Dunn JD, Allison J. The detection of multiply charged dyes using matrixassisted laser desorption/ionization mass spectrometry for the forensic examination of pen ink dyes directly from paper. J For Sci. 2007;52:120511. https://doi.org/10.1111/j.1556-4029.2007.00535.x.

33. Canamares MV, Chenal C, Birke RL, Lombardi JRA. Unified approach to Surface-Enhanced Raman Spectroscopy. J Phys Chem C. 2008;1 12:20295300. https://doi.org/10.1021/jp800167v.

\section{Publisher's Note}

Springer Nature remains neutral with regard to jurisdictional claims in published maps and institutional affiliations.

\section{Submit your manuscript to a SpringerOpen ${ }^{\odot}$ journal and benefit from:}

- Convenient online submission

- Rigorous peer review

- Open access: articles freely available online

- High visibility within the field

- Retaining the copyright to your article

Submit your next manuscript at $\boldsymbol{\nabla}$ springeropen.com 